\section{Syphilis infection in an HIV patient presenting with leukemoid reaction: Case report and review of the literature}

\author{
Athina Lioni, ${ }^{1}$ \\ Markela-Pagonitsa Zorzou, ${ }^{1}$ \\ Christina Kollia, ${ }^{2}$ Dimitrios Loulakis, ${ }^{2}$ \\ Fotini Ntziora, ${ }^{1}$ Fotini Stergiou, \\ Maria Boboli, ${ }^{1}$ Maria Chini ${ }^{1}$ \\ ${ }^{1}$ Infectious Diseases Unit, ${ }^{2}$ Internal \\ Medicine Department Red Cross \\ Hospital Korgialeneio Benakeio, Athens, \\ Greece
}

\begin{abstract}
Leukemoid reaction (LR) is an uncommon though dreadful sign for the treating physician, as it is related to increased mortality. In the few series that have addressed its incidence and clinical significance, infectious causes count for about half of the cases of LR, the rest accounting for cancer, drugs or rarer causes. In the HIV setting, it represents an even rarer event, owing probably to the impaired granulocytic response of AIDS patients to bacterial agents. However no report exists as to the incidence of LR to the immune-restored HIV patients adequately treated with antiretroviral therapy (ART). Syphilis is a well known cause of mild lymphocytosis, though only one report of LR exists in the congenital setting. We hereby report a case of an HIV patient adequately treated with ART, who presented with LR with a lymphomonocytic preponderance after infection with treponema pallidum.
\end{abstract}

\section{Introduction}

In the adult population, the term leukemoid reaction (LR) is used to describe an increase of the number of white blood cells (WBC) to more than $30,000-50,000 / \mathrm{mL}$ provided that all malignant hematological causes of leucocytosis have been excluded. ${ }^{1-4}$ Despite its rarity, it is a complex medical situation, where a quick diagnostic evaluation along with prompt therapeutic interventions need to be undertaken. On the top of the long list in the differential diagnosis, infectious causes, mainly of bacterial origin, dominate. Although syphilis is a well known cause of mild lymphocytosis, ${ }^{5}$ the manifestation of leukemoid reactions secondary to syphilitic infection has, to our knowledge, never before been reported in the literature.

In our case we present the case of a patient presenting with leukemoid reaction attributed to syphilis infection and we try to clarify the clinical importance of leukemoid reaction in the immunocompromised human immunodeficiency virus (HIV)infected population, where leukemoid reaction is an even rarer event.

\section{Case Report}

A 43-year old male patient was admitted in our hospital due to fever, chills and headache. He had been well until one month ago, when he had noticed a painless ulcer on the prepuce. A few days later, while the ulcer was healing, he experienced fever and fatigue. Ten days before admission he had noticed a disseminated maculopapular rash (Figure 1). He reported multiple unprotected sexual contacts during the last three months.

Concerning his medical history and way of life, the patient was homosexual (MSM) and had been diagnosed HIV positive a decade ago. He was treated with abacavir, lamivudine, darunavir, ritonavir and dolutegravir on a regular basis and had not received any other medication during the last month. His viral load was undetectable and his CD4 count was 1144 cells $/ \mu \mathrm{L}$. He did not have a history of urticaria or any other allergic reaction in the past. One year before, he had presented with maculopapular rash of the palms and soles along with hearing impairment and a high VDRL titer in the serum. He was suspected to have otosyphilis and was treated with intravenous penicillin-G 24 million units/day for 14 days as an inpatient. He had a full clinical and serologic response. All blood and CSF results are shown in Table 1.

On admission the patient was affected and ill-appearing on clinical examination. No signs of dehydration could be identified. There was a remarkable, although painless, enlargement of the inguinal, axillary and cervical lymph nodes, with a maximum diameter of $3 \mathrm{~cm}$. A maculopapular rash involving the trunk and the extremities was present. Despite a headache, neither nuchal rigidity, nor Kernig or Brudzinsky signs were present.

On complete blood count (CBC), an extreme leukocytosis (WBC: 63,300/mL) was shown, with a lymphomonocytic prevalence. Blood nitrogen (BUN), creatinine and urine specific gravity were within normal limits. On cerebrospinal fluid (CSF) there were 19 WBC (95\% lymphocytes). CSF glucose and protein were within normal limits. Blood cultures were repetitively
Correspondence: Markela-Pagonitsa Zorzou, 11, Patelida str, Chios 82132, Greece. Tel.: +30.2132068466 - Fax: +30.2132068466. E-mail:mpzorzou@gmail.com

Key words: HIV, syphilis, leukemoid reaction

Contributions: AL, MPZ, FZ, CK and DL contributed substantially to the conception and design of the case report submitted; $\mathrm{MB}$ and FS drafted and revised the article; MC approved the final version of the article.

Conflict of interest: the authors declare no potential conflict of interest.

Funding: none.

Received for publication: 18 October 2017.

Revision received: 21 December 2017.

Accepted for publication: 9 January 2018

This work is licensed under a Creative Commons Attribution-NonCommercial 4.0 International License (CC BY-NC 4.0).

(C) Copyright A. Lioni et al., 2018

Licensee PAGEPress, Italy

Infectious Disease Reports 2018; 10:7410

doi:10.4081/idr.2018.7410

negative (Table 1).

Based on blood and CSF results, the patient was treated for neurosyphilis with 24 million units of intravenous penicillin $\mathrm{G}$ for 11 days. Despite an extensive work-up no other cause of leukocytosis, either infectious, or hematological or neoplastic could be identified. Leukocytosis gradually subsided, along with fever and rash. Two weeks later, the patient had fully recovered and was discharged. At his regular follow-up visit, three months later, he remained in excellent condition, with no residual symptoms.

\section{Discussion}

Leukemoid reaction represents a rather uncommon finding, with an incidence of less than $1 \%$ in the hospitalized population. ${ }^{1}$ The lack of a uniform value beyond which leukocytosis is considered abnormal hinders the comparison of data among different studies. In the adult population, an arbitrary value of 30,000 WBC per milliliter has been adopted. ${ }^{1}$ However, lower ${ }^{2,3}$ and higher ${ }^{4-6}$ values have also been used as a cut-off value. A polymorphonuclear type of LR (so called reactive neutrophilia) is often encountered in several settings, mainly in bacterial and mycobacterial infections and in solid tumors. However other infrequent causes such as intoxications, severe bleed- 
ing or hemolysis have also been implicated. A lymphocytic or lymphomonocytic type of LR is, most commonly, the result of viral infections (with Epstein-Barr virus accounting for the majority of them), but has also been attributed to bacterial infections, like pertussis, or mycobacterial infections, mainly tuberculosis. ${ }^{5}$

The true meaning of such a finding has been recently elucidated in a few large series. In the general population, LR represents an exaggerative response to infection in almost half of the cases, ${ }^{1,2}$ with sepsis, lower respiratory and urinary tract infections predominating. When lower cut-off values are utilized, infectious causes are even more common (up to $75 \%$ ). ${ }^{3}$ However, in the other half of the cases, the final diagnosis is other than infection, most often malignancies, or drugs (glucocorticoids, granulocyte-stimulating factors). In a small percentage of cases, even rarer causes can be identified, such as bleeding, major operations, pancreatitis or vaginal delivery. ${ }^{1,4}$

In the oncologic population, LR often accompanies G-CSF or glucocorticoid administration in more than half of the patients. ${ }^{6}$ Only in $15 \%$ of patients LR represents true infection. ${ }^{6}$ In another $10 \%$ of the oncologic population, a term called paraneoplastic LR was adopted to describe those patients whose LR was finally attributed to a large tumor burden and tumor involving most commonly the lung. Indeed, the association of tumor with increased myelopoiesis is the result of the accumulation of various, immature, myeloid cells characterized by their ability to suppress host anti-tumor immunity and enhance tumor expansion. These cells, representing less than $1 \%$ of the mononuclear cells in healthy humans, greatly expand to more than $50 \%$ of the mononuclear cells in metastatic disease. Given their strong association with tumor expansion, they have become an attractive therapeutic target.?

While infection-related LR represents less than half of the cases, LR is still considered an alarming sign. Indeed, advanced age and the presence of infection and sepsis seem to predict in-hospital mortality in a statistically significant manner. Two points are worth underlining in the infection related cases. The first is that the duration of LR rather than the peak of the LR seems to influence survival. In fact, in-hospital mortality was extremely higher to those patients, whose LR lasted for more than 24 hours, probably reflecting aggressive infectious agents, defective immune responses or inadequate treatment. The second is that the presence of LR has been statistically correlated with the presence of positive blood cultures or of clostridium toxin, necessitat- ing a thorough evaluation towards these two parameters to every patient with LR. ${ }^{1}$

Discriminating those patients in whom an infectious cause is involved and who would mostly benefit from the administration of antimicrobial agents, is a rather difficult task in the clinical practice. Most series agree that the majority of patients with LR are at first considered to be septic and treated likewise on admission, resulting thus in antibiotic overuse. Still, there is no consensus as to the findings that would point towards an infectious cause. In the series studied the presence of fever seems to represent a strong predictor of infection. ${ }^{2}$ Extreme LR (more than 45,000 WBC) is rather related to non-infectious, most commonly malignant causes. ${ }^{2}$ When infection is the cause of LR, pneumonia, urinary tract infections, sepsis and Clostridium difficile enterocolitis predominate. ${ }^{1,2}$ Indeed, the strong association of leukocytosis with Clostridium spp, as been widely recognized, either signaling more severe disease, as in the case of Clostridium difficile infections, or accompanying severe puerperal delii. ${ }^{1}$ Based on the above principles, a risk stratification index could be designated in a prospective manner.

In the HIV setting the presence of LR is even more uncommon event, probably reflecting the impaired granulocytic response of these patients. ${ }^{8,9}$ However, few cases of LR in HIV patients have been reported in the literature namely two patients with Clostridium difficile infection ${ }^{10}$ and one patient with MAC infection after antiretroviral treatment (ART) initiation, probably reflecting immune reconstitution inflammatory syndrome. ${ }^{11}$ What difinfections as in the case of Clostridium sor-

ferentiates our case from the previous reported ones, is the fact that our patient was immunologically competent, which probably facilitated the exaggerated bone marrow response to the infectious agent. After the introduction of potent active antiretroviral therapy worldwide, resulting in immune restoration of $\mathrm{HIV}$-infected patients, such responses may become more common in the future and may well be triggered by unusual infectious agents as in our case. However, the inciting infectious agents reported above, do not differ from those reported in the non-HIV population, further reinforcing thus, the strong association of LR with specific agents, such as Clostridium species infections..$^{1-4}$

Syphilis is a well recognized bacterial disease, known to present with mild lymphocytosis. ${ }^{5}$ To our knowledge, there has been no report of LR in correlation with syphilis, either in the primary, secondary or tertiary setting of the disease, with the exception of a report of a case of congenital syphilis. ${ }^{12}$

Leukemoid reaction is considered a medical emergency for the treating physician. Even though half of the cases of LR are likely due to infections, the astute physician is called to discriminate those cases that would benefit from early antimicrobial treatment, whilst withholding inappropriate antimicrobial overuse. In the HIV population, such an approach is even more stressful, given the impaired immune status of these patients. However, the same principles in the differential diagnosis must be taken into account. A comprehensive approach, with a focus to the most common causes of LR is mandated, with a high index of suspicion for sepsis, Clostridium difficile

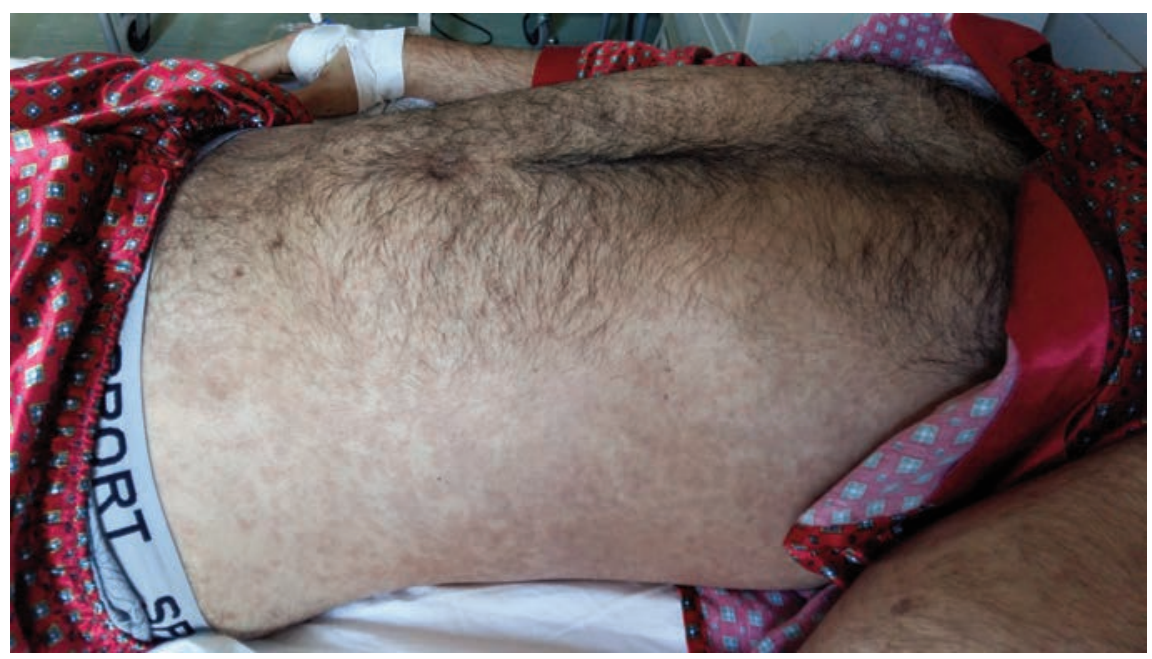

Figure 1. Patient on admission. 
enterocolitis, urinary and respiratory tract infections. Blood cultures should always be endorsed in the diagnostic procedure, based on the strong association of positive blood cultures to LR. Other clinical and laboratory features such as fever and other signs of infections must be taken into account. Noninfectious causes must be always included in the differential diagnosis and clues to other non-infectious etiologies must also be sought. The rationale to initiate antimicro- bial treatment must be based on the combination of the clinical judgement with these findings as, till now, no well documented work-up exists. This was also the case of our patient who, apart from LR, had a typical clinical picture of syphilis and consistent history. These additional clues led to the timely and specific initiation of antimicrobial treatment while other possible causes, were also considered.

\section{Conclusions}

Leukemoid reaction is an alarming feature for the treating physician. Sepsis, clostridium difficile enterocolitis, respiratory and urinary tract infections are common causes of LR. In the HIV setting, even though a rarer event, the differential diagnosis includes the same agents. Syphilis, has never before been reported as a cause of

Table 1. Analyses panel.

\begin{tabular}{|c|c|c|c|c|c|}
\hline & 1 year before & On admission & Day 7 & Day 11 & Day 60 (follow-up) \\
\hline Hematocrit (\%) & 39.1 & 38.2 & 32.7 & 35.4 & 39.4 \\
\hline Hemoglobulin (g/dL) & 12.8 & 12.9 & 11.3 & 11.8 & 13.5 \\
\hline White blood cells $(\times 109 / \mathrm{L})$ & 8.9 & 63.3 & 14.1 & 10.3 & 9.7 \\
\hline Polymorphonuclear (\%) & 65 & 20.6 & 39.3 & 29 & 41 \\
\hline Lymphocytes (\%) & 25 & 49.5 & 54.4 & 58 & 39 \\
\hline Monocytes (\%) & 6 & 29.5 & 11.9 & 8.3 & 10 \\
\hline Platelets ( L) & 383,000 & 320,000 & 509,000 & 457,000 & 415,000 \\
\hline Erythrocyte Sedimentation Rate (mm/1st hr) & 43 & 45 & & & 28 \\
\hline Glucose (mg/dL) & 86 & 79 & 91 & 71 & 75 \\
\hline Blood Urea Nitrogen (mg/dL) & 17 & 12 & 9 & 15 & 15 \\
\hline Creatinine (mg/dL) & 1.1 & 0.9 & 0.7 & 0.8 & 0.8 \\
\hline Sodium (mmol/L) & 137 & 121 & 136 & 137 & 138 \\
\hline Potassium (mmol/L) & 4.3 & 3.8 & 4.3 & 4.5 & 4.4 \\
\hline Calcium (mg/dL) & & 7.1 & 8.5 & & \\
\hline Bilirubin (mg/dL) & & 0.46 & 0.29 & 0.24 & \\
\hline Aspartase Transferase (U/L) & 14 & & 22 & 17 & \\
\hline Alanine Tranferase (U/L) & 19 & 68 & 31 & 34 & \\
\hline Y-Glutamyltranferase $\gamma(\mathrm{U} / \mathrm{L})$ & 22 & 63 & 24 & 26 & \\
\hline Creatinophosphokinase (U/L) & & 231 & 40 & 28 & \\
\hline Alkaline Phosphatase (U/L) & & 88 & 56 & 78 & \\
\hline Lactate Dehydrogonase (U/L) & 232 & & 228 & 175 & \\
\hline Total protein $(\mathrm{g} / \mathrm{L})$ & 64 & 65 & & & \\
\hline Albumin $(\mathrm{g} / \mathrm{L})$ & 35 & 25 & & & \\
\hline Globulin $(\mathrm{r} / \mathrm{L})$ & 29 & 40 & & & \\
\hline C-Reactive Protein $(<0.5)$ & 0.8 & 53.1 & 3.1 & 1.8 & 0.4 \\
\hline International Normalized Ratio & & 1.05 & & & \\
\hline Activated partial thromboplastin time & & 31 & & & \\
\hline Fibrinogen (mg/dL) & & 512 & & & \\
\hline HIV RNA (copies/mL) & Non detectable & Non detectable & & & Non detectable \\
\hline CD4 (cells/UL) & 1088 & 1144 & & & 1096 \\
\hline Rapid plasma regain (serum) & $1 / 31$ & $1 / 1024$ & & & $1 / 32$ \\
\hline Venereal Disease Research Laboratory (serum) & $1 / 256$ & $1 / 128$ & & & \\
\hline VDRL (CSF) & Not determined* & Negative & & & \\
\hline Cerebrospinal fluid white blood cells & 12 (100\% lymphocytes) & 19 (95\% lymphocytes) & & & \\
\hline CSF glucose (mg/dL) & 56 & 62 & & & \\
\hline CSF protein $(\mathrm{mg} / \mathrm{dL})$ & 34 & 27 & & & \\
\hline Procalcitonin & & 0.59 & & & \\
\hline Blood Cultures & & Sterile & Sterile & & \\
\hline
\end{tabular}

*Inappropriate sample. 
LR, except from one report in congenital syphilis. Uncommon causes, such as syphilis, must be included in the differential diagnosis of LR. Larger series of patients with LR in the future may better define the clinical or laboratory findings that point to an infectious etiology and facilitate the diagnostic procedure.

\section{References}

1. Potasman I, Gruper M. Leukemoid reaction: spectrum and prognosis of 173 adult patients. Clin Inf Dis 2013;57:177-81.

2. Reding MT, Hibbs JR, Morrisson VA, et al. Diagnosis and management of 100 consecutive patients with extreme granulocytic leukocytosis. Am J Medicine 1998;104:12-8.

3. Lawrence WR, Raveh DD, Rudensky BB, Munter GG. Extreme leukocytosis in the emergency department. QJM 2007;100: 217-23.

4. Sakka V, Tsiodras S, GiamarellosBourboulis E, Giamarellou H. An update on the etiology and diagnostic evaluation of a leukemoid reaction. Eur J Intenal Medicine 2006;17:394-98.

5. George TI. Malignant or benign leukocytosis. Hematology 2012;475-84.

6. Granger JM, Kontoyiannis DP. Etiology and outcome of extreme leukocytosis in 758 nonhematologic cancer patients. A retrospective single-institution study. Cancer 2009;3919-23.

7. Wilcox RA. Cancer-Associated Myeloproliferation: old association, new therapeutic target. Mayo Clin Proc 2010;85:656-63.

8. Perkocha LA, Rodgers GM. Hematologic aspects of human immunodeficiency virus infection: laboratory and clinical considerations. Am J Hematology1988;29:94-105.

9. Kuritzkes DR. Neutropenia, neutrophil dysfunction and bacterial infection in patients with human immunodeficiency virus disease: The role of granulocytecolony stimulating factor. Clin Inf Dis 2000;30:256-60.

10. De Toledo FGS, Symes SN. Leukemoid reaction due to Clostridium difficile infection in acquired immunodeficiency syndrome: two case reports and a review of the literature. South Med J 2004;97:388-92.

11. Brown M, Williams IG, Miller RF. Deterioration of disseminated cutaneous Mycobacterium Avium complex with a leukaemoid reaction following institution of highly active antiretroviral therapy. Sex Transm Infect 2001;149150.

12. Karayalcin G, Khaniju A, Kim KY et al. Monocytosis in congenital syphilis Am J Dis Child 1977;131:782-3. 